\title{
Prognostic Model Built on Blood-based Biomarkers in Patients with Metastatic Colorectal Cancer
}

\author{
Wen-Zhuo He ${ }^{1,2,3}$, Chang Jiang ${ }^{1,2,3}$, Chen-Xi Yin ${ }^{1,2,3}$, Gui-Fang Guo ${ }^{1,2,3}$, Ru-Ming \\ Rong $^{1,2,3}$, Hui-Juan Qiu ${ }^{1,2,3}$, Xu-Xian Chen ${ }^{1,2,3}$, Bei Zhang ${ }^{1,2,3}$, Liang-Ping Xia ${ }^{1,2,3 *}$
}

\begin{abstract}
Background: We had previously showed that the neutrophil lymphocyte ratio (NLR), $\gamma$-glutamyl transpeptidase (GGT) and carcinoembryonic antigen (CEA) are prognostic factors for metastatic colorectal cancer (mCRC) patients. In this study we developed a prognostic model based on these three indices. Materials and Methods: A total of 243 patients who were initially diagnosed as mCRC between 2005 and 2010 in the Sun Yat-sen University Cancer Center were studied. The endpoint was overall survival (OS). Results: NLR>3, elevated GGT and elevated CEA were confirmed as independent risk factors which could predict poor prognosis. Patients could be divided into three groups according to the number of risk factors they had. Those with two or three were defined as the high risk group, individuals with one risk factor as the modest risk group and patients without risk factor as the low risk group. The OS values for these three groups were 16.2 months (2.80 68.8), 24.2 months (4.07 79.0), and 37.2 months $(12.6 \sim 87.8)$, respectively $(p<0.001)$. Conclusions: We developed a simple but useful model based on NLR, GGT and CEA to provide prognostic information to clinical practice in highly selected mCRC patients. Further prospective and multi-center studies are warranted to test our model.
\end{abstract}

Keywords: GGT - CEA - NLR - colorectal cancer - prognosis model

Asian Pac J Cancer Prev, 15 (17), 7327-7331

\section{Introduction}

Metastatic colorectal cancer (mCRC) patients yield distinct prognosis (Ozdemir et al., 2014), and many efforts had been done to distinguish those patients. For example, mircoRNAs, such as miR-21 (Tokarz and Blasiak, 2012), various kinds of proteins including hypoxia induced factor (HIF) (Cao et al., 2009; Baba et al., 2010) and vascular endothelial growth factor (VEGF) (Noike et al., 2008), circulating tumor cells (CTCs) (Cohen et al., 2008) and other factors had been studied as correlated with prognosis. However, some of the studies required advanced equipments and technologies (Cohen et al., 2008), some of the studies were depended on special tested objects which were not routinely available. Thus their clinical uses were limited, especially in developing countries and primary hospitals.

In recent years, more and more evidences had shown that some indexes which were contained in the routine tests, such as blood routine test and biochemical test, could also successfully group patients regarding to their prognosis. For example, Chiang reported that neutrophil lymphocyte ratio (NLR) more than 3 was a predictor for poor prognosis (Chiang et al., 2012), and platelet lymphocyte ratio (PLR) was also reported as prognostic factor (Kwon et al., 2012; Kemal et al., 2014). $\gamma$-glutamyl transpeptidase (GGT), alkaline phosphatase (ALP), lactate dehydrogenase $(\mathrm{LDH})$ which were included in biochemical test, were also showed as prognostic factors (Saif et al., 2005; Koukourakis et al., 2006; Pompella et al., 2006; Franzini et al., 2008; Franzini et al., 2009; Xie et al., 2014). However, previous studies had several drawbacks. First, they included patients with various stages, varying from stage II to stage IV (Farias et al., 2011), second, most of those studies only evaluated one index without considering others (Saif et al., 2005; Koukourakis et al., 2006).

To overcome the two shortages, we focused on mCRC patients who accepted standard chemotherapy. Our work showed that NLR, GGT and CEA were independent prognostic factor (He et al., 2013; He et al., 2013). And the combination of NLR and CEA, GGT and CEA were better than each of them alone. So, we speculated that the combination of the three indexes would be better than two. We further evaluated whether or not a prognostic model could be build based on these three factors, which could be widely applicable since NLR, GGT and CEA are routinely available. 


\section{Materials and Methods}

\section{Patient selection}

The inclusion criteria were as follows: 1) patients who were diagnosed as metastatic colorectal cancer initially between 2005 to 2010 at Sun Yat-sen university cancer center; 2) patients who were with Eastern Cooperative Oncology Group (ECOG) status $\leq 2 ; 3$ ) patients whose records of blood routine test, biochemical test and CEA before chemotherapy were available; 4) patients whose follow-up information were available. Patients were excluded if med the following criteria: 1) patients who were with infection or hematology disease at the first time presented in our cancer center; 2) patients who were with hyperpyrexia and intestinal obstruction at the first time presented in our cancer center; 3) patients who were with liver disease including alcoholic hepatitis and $\mathrm{HBV}$ or $\mathrm{HCV}$ infection at the first time presented in our cancer center.

\section{Laboratory measurement}

Neutrophil and lymphocyte counts were contained in blood routine test while GGT was contained in biochemical test. Sysmex XE-5000 ${ }^{\mathrm{TM}}$ Automated Hematology System (Shanghai, China) was used to conduct blood routine test and Hitachi Automatic Analyzer 7600-020 (Tokyo, Japan) was used for biochemical test. Roche Elecsys 2010 Chemistry Analyzer (Basel, Switzerland) was used to evaluate CEA.

\section{Calculation and grouping of NLR, GGT and CEA}

NLR was calculated by dividing the neutrophil by the lymphocyte count, and it was categorized into two groups (>3 and $\leq 3$ ) (Chiang et al., 2012). GGT was divided into two groups as $>50$ (increased) and $\leq 50$ (normal) based on the normative reference while CEA was divided as $>5$ (increased) and $\leq 5$ (normal).

\section{Patient follow-up and statistical analysis}

The primary endpoint of this study was overall survival (OS), defining as the time from diagnosis to the date of death or the last date of follow-up, which was conducted at $31^{\text {st }}$ March 2013. Progress-free survival (PFS) of first line chemotherapy, defined as the time from initiating first-line chemotherapy to disease progression, was also examined in this study.

SPSS version 13.0 was used to perform statistical analyses. Patient characteristics were compared by frequencies and descriptive statistics. The Kaplan-Meier method was used to calculate survival curves, and loge-rank tests were used to compare the differences. Cox proportional hazards model (multivariate analysis) by backward elimination of insignificant explanatory variables was used to test independence. A P value equals to 0.05 or less was considered to be significant.

\section{Results}

\section{Patient characteristics}

We had updated our database at March 2013. 243 patients were included, among which 155 were male and
88 were female. Median OS was 21.5 months (range from 2.8 months to 87.8 months). The detailed information was showed in our previous work (He et al., 2013).

\section{$N L R, G G T$ and CEA were all independent prognostic factors.}

In our previous works ${ }^{14,15}$, NLR and CEA, GGT and CEA were independent prognostic factors, while age, gender, PLR, ALP and LDH were not. So, here, we first put the three factors into multivariate analysis together. The result turned out to be that all of the NLR $(\mathrm{HR}=1.32$, $p=0.04)$, CEA $(\mathrm{HR}=2.06, p<0.001)$ and GGT $(\mathrm{HR}=1.84$, $p<0.001)$, were independent.

\section{NLR and GGT served as supplement to each other.}

NLR could further divide patients on the basis of CEA and GGT, as shown in Table 1. In our precious research which studied CEA and GGT, we found patients' OS was significant different among groups with none, one and both of increased indexes. Now, once NLR was taken into account in each group, the patients' OS were further successfully distinguished. For example, among patients who were with normal CEA and GGT levels (37 patients), the median OS of patients who were with $\mathrm{NLR} \leq 3$ (29 patients) was 37.2 months (range from 12.6 to 87.8 months); the other 8 patients were with NLR $>3$ and their median OS was 16.8 months (range from 13.8 to 70.0 months). Other detailed date was shown in Table 2. Similarly, GGT could also divide patients on the basis of CEA and NLR, as shown in Table 2.

Table 1. NLR Could Further Divide Patients on the Basis of CEA and GGT

\begin{tabular}{lccccc}
\hline CEA & GGT & NLR & No. of patients & OS (range) & $p$ \\
\hline normal & \multirow{2}{*}{ normal } & $\leq 3$ & 29 & $37.2(12.6 \sim 87.8)$ & 0.03 \\
& & $>3$ & 8 & $16.8(13.8 \sim 70.0)$ & \\
increased & \multirow{2}{*}{ normal } & $\leq 3$ & 82 & $25.3(4.1 \sim 79.0)$ & 0.04 \\
& & $>3$ & 37 & $14.1(3.4 \sim 68.8)$ & \\
\multirow{2}{*}{ normal } & \multirow{2}{*}{ increased } & $\leq 3$ & 11 & $26.4(4.3 \sim 73.8)$ & \\
& & $>3$ & 12 & $15.2(3.2 \sim 67.3)$ & \\
\multirow{2}{*}{ increased } & \multirow{2}{*}{ increased } & $\leq 3$ & 35 & $16.7(5.0 \sim 34.8)$ & 0.81 \\
& & $>3$ & 29 & $13.5(2.8 \sim 31.3)$ & \\
\hline
\end{tabular}

Table 2. GGT Could Further Divide Patients on the Basis of CEA and NLR

\begin{tabular}{lccccc}
\hline CEA & NLR & GGT & No. of patients & OS (range) & $p$ \\
\hline normal & $\leq 3$ & normal & 29 & $37.2(12.6 \sim 87.8)$ & 0.04 \\
& & increased & 11 & $26.4(4.3 \sim 73.8)$ & \\
normal & $>3$ & normal & 8 & $16.8(13.8 \sim 70.0)$ & 0.01 \\
& & increased & 12 & $15.2(3.2 \sim 67.3)$ & \\
increased & $\leq 3$ & normal & 82 & $25.3(4.1 \sim 79.0)$ & \\
& & increased & 35 & $16.7(5.0 \sim 34.8)$ & \\
increased & $>3$ & normal & 37 & $14.1(3.4 \sim 68.8)$ & 0.2 \\
& & increased & 29 & $13.5(2.8 \sim 31.3)$ & \\
\hline
\end{tabular}

Table 3. The Score of Each Factor

\begin{tabular}{lcc}
\hline & variable & Score \\
\hline GGT & $\leq 50$ & 0 \\
& $>50$ & 2 \\
CEA & $\leq 5$ & 0 \\
& $>5$ & 2 \\
NLR & $\leq 3$ & 0 \\
& $>3$ & 1 \\
\hline
\end{tabular}


Prognostic model based on the number of risk factors.

NLR>3, increased GGT and increased CEA were found as risk factors. We then conducted a prognostic model based on the three factors. Patients were divided into four groups according to the number of risk factors they had: $0,1,2$ and 3 . The median OS of the four groups were $37.2,24.2,18.1$ and 13.46 months $(p<0.001)$. The

A

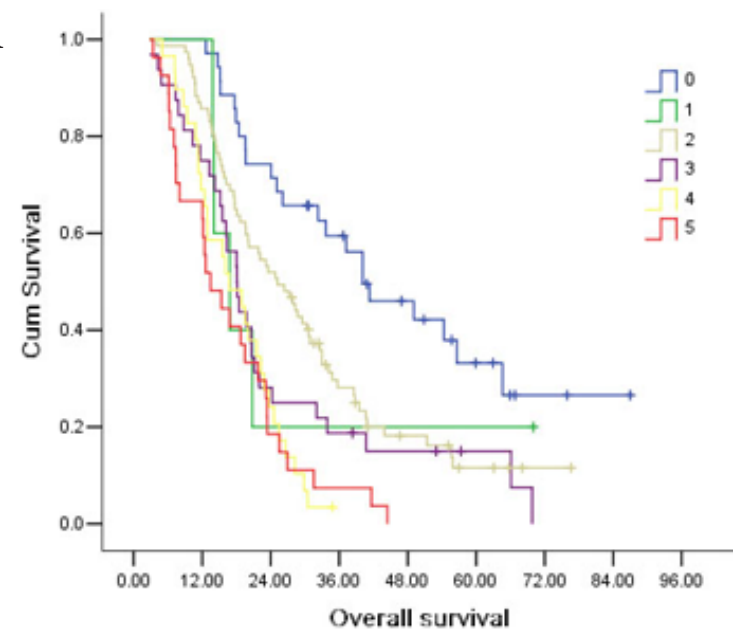

B

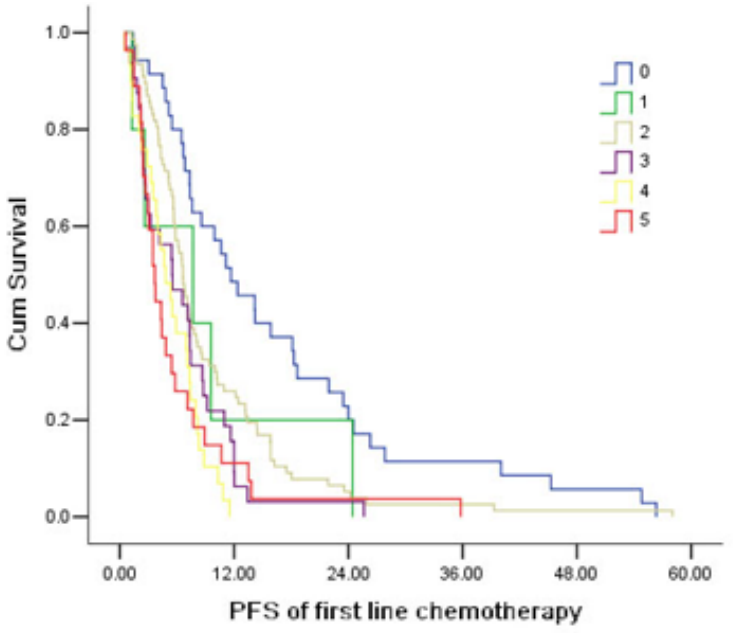

Figure 1. OS (A) and PFS (B) of Patients Grouped by Relative Risk Score

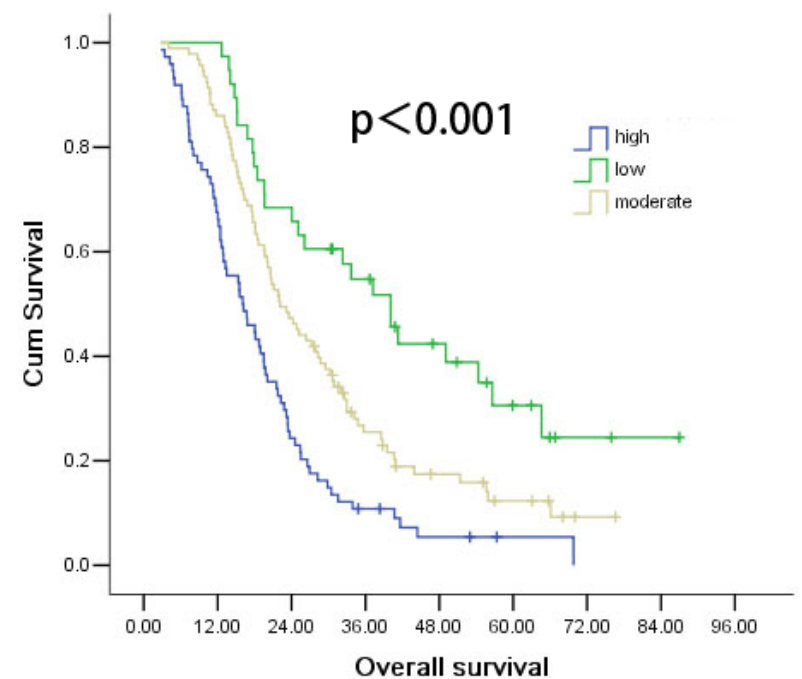

Figure 2. OS of Patients in High Risk Group, Modest Risk Group and Low Risk Group median PFS of the four groups were 11.7, 6.3,4.9 and 3.7 months $(p<0.001)$.

\section{Prognostic model based on the relative risk of prognostic} factors.

In multivariate analysis the three factors were with different relative risk called hazard ratio. We then evaluate whether hazard ratio could pose different impact on prognosis. The hazard ratio of GGT and CEA were almost 2 while the hazard ratio of NLR was almost 1 . We scored the GGT, CEA and NLR according to their hazard ratio, as shown in Table 3. The total score of one patient was calculated as the sum of the score on GGT, CEA and NLR. $\mathrm{OS}$ and PFS of patients grouped by relative risk score were shown in Figure 1.

The differences between dividing patients according to number of risk factors and according to relative risk had two aspects. First, when patients were with only one risk factor, they might be scored one (NLR $>3$ ) or two (increased GGT or CEA). Second, when patients were with two risk factors, they might be scored three (NLR $>3$ and increased GGT, or NLR $>3$ and increased CEA ) or four (increased GGT and CEA). So we next evaluated whether there was any difference between patients who scored one and two, and any difference between patients who scored three and four. Median OS of patients scored one were 22.8 months (range from 13.8 to 70.0 months) and patients scored two were 23.2 months (range from 4.1 to 76.0 months), $p=0.77$. Median OS of patients scored three were 18.1 months (range from 3.2 to 69.8 months) and patients scored four were 16.7 months (range from 5.0 to 34.8 months), $p=0.26$. Based on our limited date, relative risk was not superior to number of risk factors.

\section{Optimizing of the prognostic model}

So, we decided to divide patients according to number of risk factors. We next evaluated whether the grouping could be optimized or not. We found that the survival curves of patients with two and three risk factors were closed to each other. As shown in Table 2 and 3, when patients were with increased GGT and CEA, NLR could not further divide patients and when patients were with increased CEA and NLR $>3$, GGT also cannot divide patients. Thus, we defined that patients who were with two or three risk factors were high risk group, patients who were with one risk factor were modest risk group and patients with no risk factor were low risk group. The OS of high risk, modest risk and low risk patients were 16.2 months (2.8 68.7), 24.2 months (4.1 79.0), 37.2 months (12.6 87.8), respectively. The results were shown in Figure 2.

\section{Discussion}

In the current study, we found that NLR, GGT and CEA were independent prognostic factors when considered together, and NLR can identify patients with poor prognosis based on the combination of GGT and CEA, the same ability of GGT was also found. These findings suggest that the combination of the three indexes may be better than the combination of two of them. We 
further tried to establish a prognostic model by combining the three indexes together. Firstly, the patients were divided into four groups in terms of the index number involved. However, the survival curves of patients with two and three indexes overlapped. Secondly, we studied the hazard ratio of each index, which may reflex the extent when they impact patients' prognosis. However, we failed to find any advantage of hazard ratio. Finally, based on the above analyses, the optimal model was established as none index, one index and two or three indexes involved, and the survival curves separated very well.

The model based on the three indexers had advantage in theory. The three factors represented different pathways: GGT was thought as correlated with cellular defense and contributing to drug resistance (Franzini et al., 2006), CEA was correlated with the invasion and metastasis of tumor cells (Goldstein and Mitchell, 2005; Yang et al., 2011) while NLR was thought as represent an unfavorable tumor microenvironment (An et al., 2010). The model based on three factors may represent a more comprehensive understanding to tumors thus was superior to two factors.

The clinic-pathological characteristics should be taken into account when develop a prognostic model since they are basic and easily obtained while with predict values (Shiono et al., 2006). In our study, most of the clinicpathological characteristics were taken into account. Firstly, all significant clinicopathological characteristics in the univariate analysis in our previous studies were put into the multivariate analysis (He et al., 2013; He et al., 2013). Apart from NRL, GGT and CEA, the traditional clinic-pathological characteristics were not independent. The consistency might be caused by the criterions of selecting patients in our study, since all patients were initially diagnosed as mCRC, and accepted standard chemotherapy. Another reason might be that most of the common clinic-pathological characteristics were balanced in each of NLR, GGT and CEA group.

We believe this model is helpful for oncologists since all of NLR, GGT and CEA are routinely available, especially in developing countries and rural hospitals, where advanced equipments and technical support are limited. However, several drawbacks of this study should taken into account if be of clinical implication. The pathological features, such as vascular invasion and perineuronal invasion, are also easy to access and with prognostic values (Shiono et al., 2006). However, those factors were not included in this paper because the patients evolved in our study were patients initially diagnosed as metastatic disease. Most of them only accepted biopsy which may result in the loss of those pathological information. This study was retrospective study and was based on one single cancer center. The specificity of GGT and NLR were limited since GGT could also increase in other disease and neutrophil might be affected by various inflammatory reactions. We had exclude patients who were with liver disease and intestinal obstruction at the first time presented in our cancer center in order to limit the bias (He et al., 2013; He et al., 2013). Franzini had reported that there were at least four kinds of GGT, and b-GGT was produced other than liver (Franzini et al., 2008; Franzini et al., 2009), thus the subtypes of GGT may improve the specificity and severe as promising candidates in the further.

In conclusion, we developed a simple but useful model to provide valuable prognostic information to clinical practice in highly selected mCRC subgroup of patient population. Further prospective and multi-center study is expected to test our model.

\section{Acknowledgements}

This work was supported by the Science and Technology Planning Project of Guang dong Province, China (2011B061300069) and the National Natural Science Foundation of China (81272641 and 81071872).

\section{References}

An X, Ding PR, Li YH, et al (2010). Elevated neutrophil to lymphocyte ratio predicts survival in advanced pancreatic cancer. Biomarkers, 15, 516-22.

Baba Y, Nosho K, Shima K, et al (2010). HIF1A overexpression is associated with poor prognosis in a cohort of 731 colorectal cancers. Am J Pathol, 176, 2292-301.

Cao D, Hou M, Guan YS, et al (2009). Expression of HIF-1 alpha and VEGF in colorectal cancer: association with clinical outcomes and prognostic implications. BMC Cancer, 9, 432 .

Chiang SF, Hung HY, Tang R, et al (2012). Can neutrophil-tolymphocyte ratio predict the survival of colorectal cancer patients who have received curative surgery electively? Int J Colorectal Dis, 27, 1347-57.

Cohen SJ, Punt CJ, Iannotti N, et al (2008). Relationship of circulating tumor cells to tumor response, progression-free survival, and overall survival in patients with metastatic colorectal cancer. J Clin Oncol, 26, 3213-21.

Farias IL, Farias JG, Rossato L, et al (2011). Correlation between TBARS levels and glycolytic enzymes: the importance to the initial evaluation of clinical outcome of colorectal cancer patients. Biomed Pharmacother, 65, 395-400.

Franzini M, Corti A, Lorenzini E, et al (2006). Modulation of cell growth and cisplatin sensitivity by membrane gammaglutamyltransferase in melanoma cells. Eur J Cancer, 42, 2623-30.

Franzini M, Bramanti E, Ottaviano V, et al (2008). A high performance gel filtration chromatography method for gamma-glutamyltransferase fraction analysis. Anal Biochem, 374, 1-6.

Franzini M, Corti A, Fornaciari I, et al (2009). Cultured human cells release soluble gamma-glutamyltransferase complexes corresponding to the plasma b-GGT. Biomarkers, 14,486-92.

Goldstein MJ, Mitchell EP(2005). Carcinoembryonic antigen in the staging and follow-up of patients with colorectal cancer. Cancer Invest, 23, 338-51.

He W, Yin C, Guo G, et al (2013). Initial neutrophil lymphocyte ratio is superior to platelet lymphocyte ratio as an adverse prognostic and predictive factor in metastatic colorectal cancer. Med Oncol, 30, 439.

He WZ, Guo GF, Yin CX, et al (2013). Gamma-glutamyl transpeptidase level is a novel adverse prognostic indicator in human metastatic colorectal cancer. Colorectal Dis, 15, 443-52.

Kemal Y, Yucel I, Ekiz K, et al (2014). Elevated serum neutrophil to lymphocyte and platelet to lymphocyte ratios could be useful in lung cancer diagnosis. Asian Pac J Cancer Prev, 15, 2651-4.

Koukourakis MI, Giatromanolaki A, Sivridis E, Gatter KC, Harris AL (2006). Lactate dehydrogenase 5 expression in 
operable colorectal cancer: strong association with survival and activated vascular endothelial growth factor pathway--a report of the tumour angiogenesis research group. J Clin Oncol, 24, 4301-8.

Kwon HC, Kim SH, Oh SY, et al (2012). Clinical significance of preoperative neutrophil-lymphocyte versus plateletlymphocyte ratio in patients with operable colorectal cancer. Biomarkers, 17, 216-22.

Noike T, Miwa S, Soeda J, Kobayashi A, Miyagawa S (2008). Increased expression of thioredoxin-1, vascular endothelial growth factor, and redox factor-1 is associated with poor prognosis in patients with liver metastasis from colorectal cancer. Hum Pathol, 39, 201-8.

Ozdemir Y, Akin ML, Sucullu I, Balta AZ, Yucel E (2014). Pretreatment neutrophil/lymphocyte ratio as a prognostic aid in colorectal cancer. Asian Pac J Cancer Prev, 15, 2647-50.

Pompella A, De Tata V, Paolicchi A, Zunino F (2006). Expression of gamma-glutamyltransferase in cancer cells and its significance in drug resistance. Biochem Pharmacol, 71, 231-8.

Saif MW, Alexander D, Wicox CM (2005). Serum alkaline phosphatase level as a prognostic tool in colorectal cancer: a study of 105 patients. J Appl Res, 5, 88-95.

Shiono S, Ishii G, Nagai K, et al (2006). Immunohistochemical prognostic factors in resected colorectal lung metastases using tissue microarray analysis. Eur J Surg Oncol, 32, 308-9.

Tokarz P, Blasiak J (2012). The role of microRNA in metastatic colorectal cancer and its significance in cancer prognosis and treatment. Acta Biochim Pol, 59, 467-74.

Xie Y, Wei ZB, Duan XW (2014). Prognostic value of pretreatment serum alkaline phosphatase in nasopharyngeal carcinoma. Asian Pac J Cancer Prev, 15, 3547-53.

Yang XQ, Chen C, Wang FB, Peng CW, Li Y (2011). Preoperative serum carcinoembryonic antigen, carbohydrate antigen 19-9 and carbohydrate antigen 125 as prognostic factors for recurrence-free survival in colorectal cancer. Asian Pac $J$ Cancer Prev, 12, 1251-6. 